\title{
Arrhythmogenic Right Ventricular Dysplasia and Brugada Syndrome Overlap
}

\author{
Hussein Rabah ${ }^{1}$, Ali Rabah ${ }^{2}$ \\ 1. Internal Medicine, Northwell Health, New York, USA 2. Division of Electrophysiology, Beirut Cardiac Institute, \\ Beirut, LBN
}

Corresponding author: Hussein Rabah, hussein-rabah@hotmail.com

\begin{abstract}
Arrhythmogenic right ventricular dysplasia (ARVD) and Brugada syndrome(BS) are associated with an increased risk of sudden cardiac death. Although they are described as two different entities, research suggests that they are not entirely separate. This paper presents a 55 years old male who presented for syncope. Interestingly, his electrocardiogram met the diagnostic criteria for both ARVD and BS. Subsequently, an implantable cardioverter-defibrillator (ICD) was implanted before discharge due to his high risk of sudden cardiac death. This case revealed that ARVD and BS clinical features can coexist in a single patient, and therefore supports the existence of a common pathophysiological basis of both diseases.

Categories: Cardiology, Internal Medicine

Keywords: arvd, brugada ecg pattern, overlap syndrome, ecg

\section{Introduction}

Arrhythmogenic right ventricular dysplasia (ARVD) is an inherited cardiomyopathy first described by Fontaine et al. in 1977 [1]. It is characterized by replacing the right ventricular myocardium with fatty tissue associated with ventricular dysfunction and arrhythmias [2,3]. In contrast, Brugada syndrome (BS) is a functional heart disease characterized by a typical EKG pattern of right bundle branch block and ST-segment elevations in the right precordial leads [4]. As opposed to patients with ARVD, patients with BS typically have no structural heart disease detectable by imaging or angiography $[5,6]$. However, it is generally known that there are clinical and pathophysiological similarities between ARVD and BS, but little is known about such an overlap. Therefore, in order to further understand such an overlap, we report here, a case of an overlapping state of ARVD and BS with unique electrocardiographic features.
\end{abstract}

Review began 04/13/2021 Review ended 04/13/2021 Published 04/14/2021

\section{(c) Copyright 2021}

Rabah et al. This is an open access article distributed under the terms of the Creative Commons Attribution License CC-BY 4.0., which permits unrestricted use, distribution, and reproduction in any medium, provided the original author and source are credited.

\section{Case Presentation}

A 55-year-old man presented for syncope. Subsequently, he was admitted to the hospital for evaluation and workup. His past medical and surgical histories were unremarkable. He denied any family history of sudden cardiac death. Electrocardiogram (EKG) (Figure 1) showed right bundle branch block and ST-elevation in lead v1 consistent with Brugada type 1 EKG (Figure 2), besides an epsilon wave and T waves inversions in the right precordial leads v1, v2, v3 consistent with ARVD (Figure 2). Echocardiography showed a basal right ventricular free wall aneurysm and dyskinesis. As a result, the patient was diagnosed with ARVD after meeting the two major EKG parameters of the 2010 revised task force criteria (TFC): epsilon waves in the right precordial leads implying a depolarization abnormalities, and the inverted $\mathrm{T}$ waves in v1, v2, and v3 signifying a repolarization anomalies. His imaging findings met a single minor criterion, not enough for ARVD diagnosis. Interestingly, he also fulfilled the diagnostic criteria of BS as he had syncope in the presence of ST elevations in v1 consistent with BS EKG type 1. The patient was discharged from the hospital after the insertion of an implantable cardioverter-defibrillator (ICD) due to his high risk of sudden cardiac death. 


\section{Cureus}

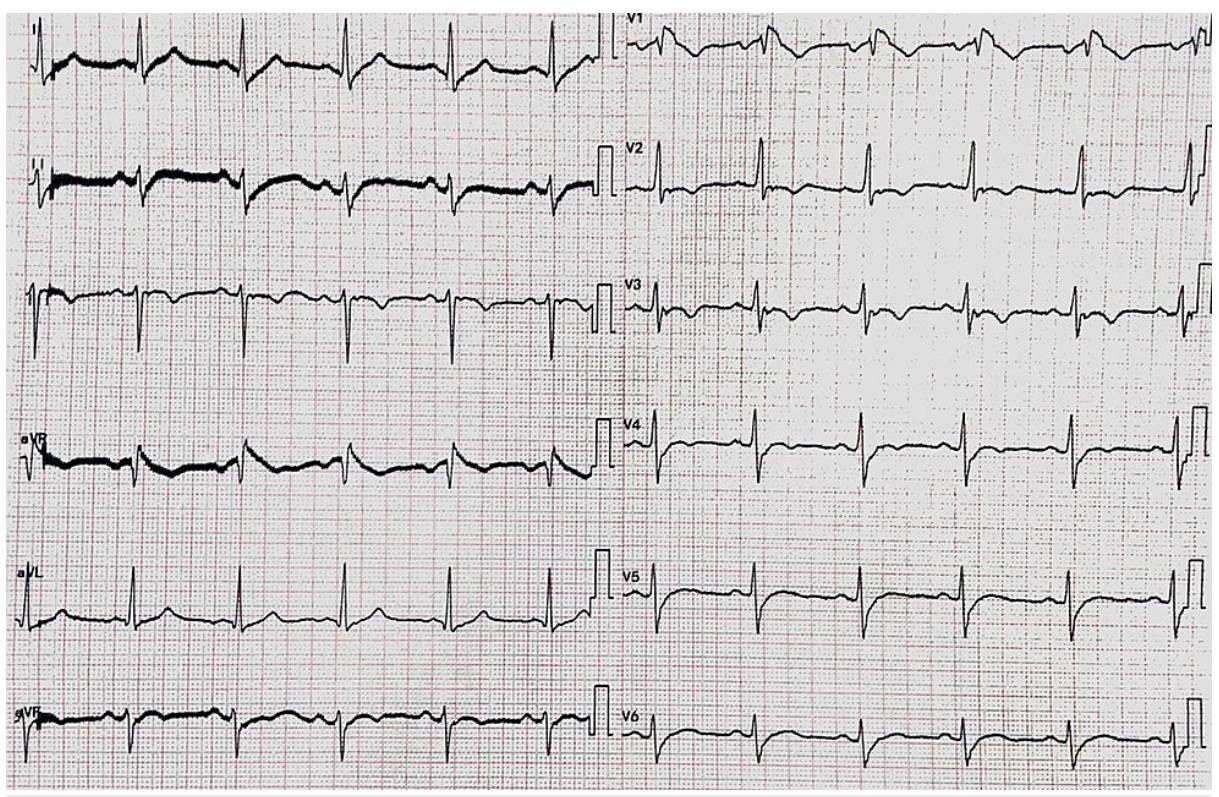

FIGURE 1: The patient's 12 lead electrocardiogram (EKG).

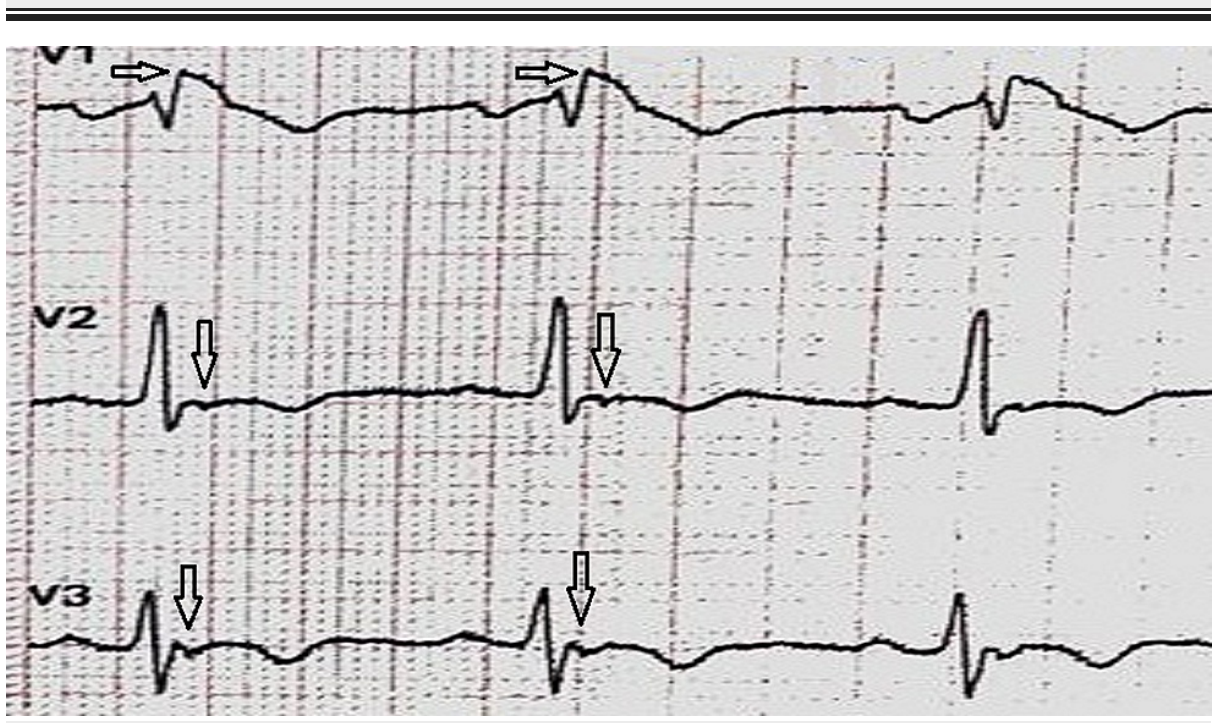

FIGURE 2: The patient's right precordial leads.

Note the ST-segment elevation in v1 consistent with Brugada type 1 EKG, the "epsilon wave" shown by the black arrows, and T wave inversion evident in the leads reflecting repolarization abnormalities.

\section{Discussion}

The patient described in this report had a unique surface electrocardiogram (EKG) (Figure 1). He met the EKG criteria for both BS and ARVD. He exhibited ST-segment elevations in V1 consistent with BS type 1 (Figure 2) [5]. Also, epsilon waves and T waves inversions were evident on the right precordial leads consistent with the diagnostic criteria of ARVD (Figure 2) [6]. Differentiating both diseases is based on the presence or absence of clinical right ventricle structural abnormalities.

Regarding this patient's echocardiography, it revealed a right ventricular basal free wall aneurysm and dyskinesis. According to the 2010 TFC, those findings met only a minor criterion for ARVD diagnosis. Therefore, he was diagnosed with ARVD by fulfilling two major EKG criteria: apparent epsilon waves and $\mathrm{T}$ wave inversions in v1, v2, and v3. However, the ST-elevation in lead v1 and syncope are manifestations of an overlapping BS.

Although those diseases are described as two different entities, research supports that they are not entirely separate. Cox et al. concluded that desmosomal mutations were present in most patients with ARVD [7]. 
Interestingly, Cerrone et al. described that disruption of desmosomal integrity could alter sodium current and coincide with BS phenotype [8]. Pascual et al. experiment proposed that both ARVD and BS are diseases of connexons [9]. This supports that both diseases share a common molecular background and may explain the unique characteristics of the EKG described in this report. The molecular changes affected the myocardium's electrophysiological characteristics and were evident on the surface EKG as a double manifestation of the two diseases.

On the other hand, Martini et al. described six patients who experienced ventricular fibrillation (VF) and clinical evidence of right ventricle (RV) structural abnormalities; Brugada-like ECG patterns were recognized in three patients [10]. In 1996, Corrado et al. reported a family with Brugada-like ST-segment elevation, RV cardiomyopathic changes on echocardiography, and diagnostic histopathological features of ARVC [11]. Again, this opens the possibility that both diseases represent two poles of a shared spectrum, ultimately leading to an increased risk of sudden death. However, none of the described patients had simultaneous EKG manifestations of ARVD and BS similar to our patient.

Finally, clinical, EKG, and echocardiographic features discussed in this paper might point to an overlap syndrome between ARVD and BS. Nevertheless, such an overlap syndrome's characteristic features are still unclear, and a treatment approach to such patients is unknown. Therefore, patients with such phenotype should be identified. Due to the features of the high risk of sudden cardiac death in the patient described, including spontaneous BS type 1 EKG pattern and an episode of syncope, an ICD was implanted before discharge.

\section{Conclusions}

Patients with an overlapping ARVD/Brugada state should be identified to further clarify the pathological bases of such an entity. Besides, as the clinical features are not well established, careful inspection and analysis of EKGs in patients with ARVD or BS should be done looking for any overlapping features.

\section{Additional Information \\ Disclosures}

Human subjects: Consent was obtained or waived by all participants in this study. Conflicts of interest: In compliance with the ICMJE uniform disclosure form, all authors declare the following: Payment/services info: All authors have declared that no financial support was received from any organization for the submitted work. Financial relationships: All authors have declared that they have no financial relationships at present or within the previous three years with any organizations that might have an interest in the submitted work. Other relationships: All authors have declared that there are no other relationships or activities that could appear to have influenced the submitted work.

\section{References}

1. Fontaine G, Guiraudon G, Frank R, Vedel J, Grosgogeat Y, Cabrol C, Facquet I : Stimulation studies and epicardial mapping in ventricular tachycardia: study of mechanisms and selection for surgery. Reentrant arrhythmias. University Park Press, Baltimore; 1977. 334-350.

2. Marcus FI, Fontaine GH, Guiraudon G, Frank R, Laurenceau JL, Malergue C, Grosgogeat Y: Right ventricular dysplasia: a report of 24 adult cases. Circulation. 1982, 65:384-98. 10.1161/01.cir.65.2.384

3. Marcus FI, Fontaine G: Arrhythmogenic right ventricular dysplasia/cardiomyopathy: a review . Pacing Clin Electrophysiol. 1995, 18:1298-314. 10.1111/j.1540-8159.1995.tb06971.x

4. Brugada P, Brugada J: Right bundle branch block, persistent ST segment elevation and sudden cardiac death: a distinct clinical and electrocardiographic syndrome: a multicenter report. J Am Coll Cardiol. 1992, 15:1391-6. 10.1016/0735-1097(92)90253-J

5. Antzelevitch C, Brugada P, Borggrefe M, et al.: Brugada syndrome: report of the second consensus conference: endorsed by the Heart Rhythm Society and the European Heart Rhythm Association. Circulation. 2005, 111:659-70. 10.1161/01.CIR.0000152479.54298.51

6. Marcus FI, McKenna WJ, Sherrill D, et al.: Diagnosis of arrhythmogenic right ventricular cardiomyopathy/dysplasia: proposed modification of the task force criteria. Circulation. 2010, 121:1533-41. 10.1161/CIRCULATIONAHA.108.840827

7. Cox MG, van der Zwaag PA, van der Werf C, et al.: Arrhythmogenic right ventricular dysplasia/cardiomyopathy: pathogenic desmosome mutations in index-patients predict outcome of family screening: Dutch arrhythmogenic right ventricular dysplasia/cardiomyopathy genotype-phenotype followup study. Circulation. 2011, 123:2690-700. 10.1161/CIRCULATIONAHA.110.988287

8. Cerrone M, Delmar M: Desmosomes and the sodium channel complex: implications for arrhythmogenic cardiomyopathy and Brugada syndrome. Trends Cardiovasc Med. 2014, 24:184-90. 10.1016/j.tcm.2014.02.001

9. Agullo-Pascual E, Cerrone M, Delmar M: Arrhythmogenic cardiomyopathy and Brugada syndrome: diseases of the connexome. FEBS Lett. 2014, 588:1322-30. 10.1016/j.febslet.2014.02.008

10. Martini B, Nava A, Thiene G, et al.: Ventricular fibrillation without apparent heart disease: description of six cases. Am Heart J. 1989, 118:1203-9. 10.1016/0002-8703(89)90011-2

11. Corrado D, Nava A, Buja G, et al.: Familial cardiomyopathy underlies syndrome of right bundle branch block, ST segment elevation and sudden death. J Am Coll Cardiol. 1996, 27:443-8. 